\title{
Efficacy of Pre-Emergence and Post-Emergence Soybean Herbicides for Control of Glufosinate-, Glyphosate-, and Imidazolinone-Resistant Volunteer Corn
}

\author{
Parminder S. Chahal ${ }^{1}$, Greg Kruger ${ }^{1}$, Humberto Blanco-Canqui ${ }^{1} \&$ Amit J. Jhala ${ }^{1}$ \\ ${ }^{1}$ Department of Agronomy \& Horticulture, University of Nebraska-Lincoln, Nebraska, USA \\ Correspondence: Amit J. Jhala, Department of Agronomy \& Horticulture, University of Nebraska-Lincoln, \\ Nebraska, USA. E-mail: amit.jhala@unl.edu
}

Received: May 26, 2014 Accepted: June 13, 2014 Online Published: July 15, 2014

doi:10.5539/jas.v6n8p131 URL: http://dx.doi.org/10.5539/jas.v6n8p131

\begin{abstract}
Glyphosate-resistant corn and soybean are grown in rotations in the Midwest, including Nebraska. Volunteer corn is a problematic weed in soybean fields because it causes harvest problems, reduces yield and seed quality, and potentially harbors insects, pests, and diseases. Several pre-packaged herbicides have been registered in soybean in recent years, but response of volunteer corn to these herbicides has not yet been documented. Greenhouse experiments were conducted to evaluate the response of glufosinate-, glyphosate-, and imidazolinone-resistant volunteer corn to 20 pre-emergence (PRE) and 17 post-emergence (POST) soybean herbicides. Cumulative emergence of volunteer corn was not affected by PRE soybean herbicides compared with the nontreated control regardless of herbicide-resistant trait at 21 days after treatment (DAT). Although comparable with several other treatments, clomazone provided $\geq 90 \%$ control of glufosinate- and imidazolinone-resistant volunteer corn at 21 DAT. The POST soybean herbicides were applied when volunteer corn plants were at the 2 to 3 or 5 to 6 leaf stage. The ACCase-inhibiting herbicides, including clethodim, fenoxaprop plus fluazifop, fluazifop, quizalofop, and sethoxydim, provided $\geq 96$ and $\geq 85 \%$ control of the 2 to 3 or 5 to 6 leaf stage volunteer corn, respectively, regardless of the herbicide-resistance trait at 28 DAT. Glyphosate tank mixed with acifluorfen, chlorimuron-ethyl, or imidazolinones usually provided $>83 \%$ control of glufosinate-and imidazolinone-resistant volunteer corn when sprayed at the 2 to 3 leaf stage at 28 DAT, but control was $\leq 71 \%$ for the 5 to 6 leaf stage volunteer corn. Similar results were usually reflected in volunteer corn biomass. It is concluded that PRE soybean herbicides partially controlled volunteer corn; therefore, ACCase inhibiting herbicides are the only highly effective option for soybean growers.
\end{abstract}

Keywords: herbicide efficacy, pre-packaged herbicides, volunteer corn biomass, volunteer corn leaf stage

\section{Introduction}

The United States is the largest producer of corn in the world (USDA, 2013). In 2013, the estimated area planted to corn in the United States was about 35.39 million ha (USDA, 2013), and by 2016, this number is expected to increase to 38 million ha (Malcolm \& Aillery, 2009). Corn-soybean is the most prominent crop rotation in the Corn Belt. Glyphosate-resistant volunteer corn is a problem weed not only in soybean, but also in continuous corn rotations (Marquardt et al., 2012a). Storm damage, harvesting problems, poor stalk quality, and insect damage, among other factors, can lead to kernel and ear losses that result in volunteer corn the following year. Volunteer corn results from the overwintering of the hybrid corn used the previous year or from a failed corn stand in corn replant situation (Steckel et al., 2009; Shauck \& Smeda, 2012).

Volunteer corn was documented as a weed even before the commercialization of herbicide-resistant corn (Andersen et al., 1982; Beckett \& Stroller, 1988), with glyphosate used in rope-wick applications to control volunteer corn (Andersen et al., 1982; Beckett \& Stroller, 1988; Dale, 1981). With the commercialization of glyphosate-resistant corn and soybean in the late 1990s, growers rapidly adopted them in the Americas (Castle et al., 2006). In 2010 , more than $70 \%$ of corn and $93 \%$ of soybean planted were herbicide-resistant, primarily glyphosate-resistant (USDA-NASS, 2010). Increased adoption of glyphosate-resistant corn resulted in increasing issues of volunteer corn. Volunteer corn also plays a role in the survival and dispersal of corn rootworm and grey 
leaf spot disease; therefore, it limits the benefits of corn-soybean rotation and creates challenges for insect-resistance manage/ment (Marquardt et al., 2012b; Krupke et al., 2009; Shaw et al., 1978).

Volunteer corn is a competitive weed, as it grows taller than soybean, and like many other weeds, causes yield reduction by competing for light, space, nutrients, and moisture (Beckett \& Stoller, 1988; Marquardt et al., 2012b). In addition, if corn volunteers remain in the field until maturity, corn seeds would contaminate the harvested soybean and reduce the market quality (Deen et al., 2006). Beckett and Stroller (1988) reported 25\% soybean yield reduction at a volunteer corn density of 5,380 plants ha ${ }^{-1}$. In Minnesota, a uniform corn density of 0.4 plants $\mathrm{m}^{-1}$ of soybean row caused a 14 to $49 \%$ yield reduction depending on the location and year (Andersen et al., 1982). A recent study by Marquardt et al. (2012b) also reported a 10 to $14 \%$ soybean yield reduction. Wilson et al. (2010) reported that a volunteer corn density of 8,750 and 17,500 plants ha ${ }^{-1}$ reduced soybean yields by 10 and $27 \%$, respectively in Nebraska.

The ACCase (acetyl-coenzyme A carboxylase) inhibiting-herbicides, also known as graminicides, are often used in soybean to control grass weeds, including volunteer corn. Several studies reported that diclofop, fluazifop, quizalofop, and sethoxydim were effective for controlling volunteer corn in soybean (Andersen, 1976; Andersen et al., 1982; Andersen \& Geadelmann, 1982; Beckett \& Stroller, 1988; Beckett et al., 1992); however, the efficacy of an ACCase inhibitors can be affected by a number of factors, including the growth stage of the volunteer corn, the environmental conditions at the time of application, and the efficacy of the individual herbicide (Wilson et al., 2010). Several pre-packaged herbicide tank-mixtures have been registered in recent years and are widely used by soybean growers specifically for the control of glyphosate- and ALS inhibitor-resistant weeds.

Several PRE herbicides exist for residual grass weed control in soybean; however, none of them list volunteer corn on their labels. Information is not available, to our knowledge, in scientific literature about the response of volunteer corn to PRE soybean herbicides. In addition, several new pre-packaged herbicide tank-mixtures, such as sulfentrazone plus chloransulam-methyl (Authority ${ }^{\mathrm{TM}}$ First), sulfentrazone plus metribuzin (Authority ${ }^{\mathrm{TM}}$ MTZ), etc., have been registered for PRE weed control in soybean. These new residual herbicides may expand the weed control spectrum, though the response of herbicide-resistant volunteer corn to these herbicides is unknown. Therefore, the objectives of study were to (1) evaluate the efficacy of PRE soybean herbicides for control of glufosinate-, glyphosate-, and imidazolinone-resistant volunteer corn, and (2) evaluate the efficacy of POST soybean herbicides registered for grass weed control applied at two growth stages (2-to 3- or 4-to 5-leaf stage) for control of glufosinate-, glyphosate-, and imidazolinone-resistant volunteer corn.

\section{Materials and Methods}

\subsection{Greenhouse Experiment and Data Collection}

Greenhouse studies were conducted at the University of Nebraska-Lincoln in 2013. All PRE- and POST-applied soybean herbicides registered for grass weed control were evaluated for the control of glufosinate-, glyphosate-, and imidazolinone-resistant volunteer corn. The herbicide application rates were selected based on the recommended labeled rates. The hybrids of glufosinate-, glyphosate-, and imidazolinone-resistant corn were planted in 2012 at the South Central Agriculture Laboratory, University of Nebraska-Lincoln near Clay Center, Nebraska. Seeds were harvested in October 2012 and kept at room temperature until they were used for this study. A preliminary study was conducted to determine the germination percentage of volunteer corn seeds. The results suggested $\geq 98 \%$ germination for each herbicide-resistant trait (data not shown).

\subsubsection{PRE Herbicide Study}

The soil used in this study was collected from a field near Lincoln, Nebraska (24\% sand, $25 \%$ clay, $51 \%$ silt, and $2.7 \%$ organic matter) with known history of no herbicide usage for at least the last eight years. Ten seeds each of glufosinate-, glyphosate-, and imidazolinone-resistant volunteer corn were planted at 2- to 3-cm depth in plastic pots $(15 \mathrm{~cm}$ diameter and $15 \mathrm{~cm}$ height) filled with the soil. The pots were watered at field capacity. Herbicides were applied on the soil surface $1 \mathrm{~d}$ after planting the seeds using a chamber track bench sprayer fitted with a 8001-E nozzle (Teejet Technologies, Wheaton, IL). The experiment was laid out in a $20 \times 3$ factorial randomized complete block design with four replications. The two factors were 20 herbicide treatments (including nontreated control) and 3 herbicide-resistant volunteer corn traits (glufosinate-, glyphosate-, and imidazolinone-resistant). The day/night temperature and photoperiod of the greenhouse were $28 / 24^{\circ} \mathrm{C}$ and $14 \mathrm{~h}$, respectively, and the pots were watered as required. The PRE soybean herbicides used in this study are listed in Table 1. Herbicide rates were selected based on the recommended labeled rates for soybean.

A cumulative number of emergences of glufosinate-, glyphosate-, and imidazolinone-resistant volunteer corn 
were recorded at 7, 14, and $21 \mathrm{~d}$ after treatment (DAT). Visual estimates of control of emerged volunteer corn plants were recorded at 7, 14, and $21 \mathrm{~d}$ after treatment (DAT) based on a 0 to $100 \%$ scale, with $0 \%$ meaning no injury or control (healthy plant) and $100 \%$ meaning complete control or severe injury with no chance of plant survival. Volunteer corn plants were harvested at the base of the plant at 21 DAT and the fresh weight was recorded. The plants were kept in a paper bag, oven dried at $60{ }^{\circ} \mathrm{C}$ for $96 \mathrm{~h}$, and dry biomass weight was recorded. The experiment was repeated again for the consistency of results.

\subsubsection{POST Herbicide Study}

Three seeds each of glufosinate-, glyphosate-, and imidazolinone-resistant volunteer corn were seeded at a depth of 2 to $3 \mathrm{~cm}$ in separate plastic pots (15 cm diameter and $15 \mathrm{~cm}$ height), filled with $75 \%$ commercial potting mix (Berger BM1 potting mix, Berger Peat Moss Ltd., Quebec, Canada) and 25\% soil. Plants were thinned to two plants per pot at 7 days after emergence. The experiment was laid out in a $2 \times 18 \times 3$ factorial randomized complete block design with four replications. The three factors included two heights of volunteer corn [2- to 3-leaf stage (12 to $15 \mathrm{~cm}$ tall) and 5- to 6-leaf stage (30 to $33 \mathrm{~cm}$ tall)], 18 herbicide treatments (including a nontreated control), and three herbicide-resistant volunteer corn traits (glufosinate-, glyphosate-, and imidazolinone-resistant). Plants were watered every other day and were supplied with nutrients using fertilizer solution (Scotts Miracle-Gro Products, Inc. Marysville, $\mathrm{OH}$ ) before $5 \mathrm{~d}$ of herbicide treatment. Herbicide treatments were applied when volunteer corn plants were at the 2- to 3-leaf stage (12- to $15-\mathrm{cm}$ tall) or the 5- to 6-leaf stage (30- to 33-cm tall). Details of POST soybean herbicides used in this study are provided in Table 2. Herbicide rates used were based on recommended labeled rates for soybean. Recommended adjuvants were added to the herbicide solutions (Table 2). Treatments were applied using the same chamber track bench sprayer noted in the PRE herbicide study.

Visual estimates of control of glufosinate-, glyphosate-, and imidazolinone-resistant volunteer corn were recorded at 7, 14, 21, and 28 DAT based on a 0 to $100 \%$ scale as explained in the PRE herbicide study. Volunteer corn plants were harvested at the base of the plant at 28 DAT and the fresh weight was recorded. The plants were kept in paper bags, oven dried at $60{ }^{\circ} \mathrm{C}$ for $96 \mathrm{~h}$ and biomass weight was recorded. The experiment was repeated again for the consistency of results.

\subsection{Statistical Analysis}

Data from PRE and POST soybean herbicide studies were subjected to ANOVA using the PROC GLIMMIX procedure in SAS version 9.3 (SAS Institute Inc, Cary, NC). Before analysis, data were tested for normality with the use of PROC UNIVARIATE. Visual estimates of volunteer control, volunteer corn emergence, and biomass data were arcsine square-root transformed before analysis; however, back-transformed data are presented with mean separation based on transformed data. For PRE herbicide study, herbicide treatments and corn types were the fixed effects, while replications and experimental repeats (nested within replication) were considered random effects. For POST herbicide study, herbicide treatments, volunteer corn type, and plant heights were the fixed effects, while replications and experimental repeats (nested within replication) were considered random effects. Where the ANOVA indicated treatment effects were significant, means were separated at $\mathrm{P} \leq 0.05$ with Tukey-Kramer's pairwise comparison test. 
Table 1. Details of pre-emergence (PRE) soybean herbicides used in this study

\begin{tabular}{|c|c|c|c|c|c|}
\hline Herbicide & & Trade name & Formulation & $\begin{array}{l}\text { Rate } \\
\left(\mathrm{g} \text { ai ha }{ }^{-1}\right)\end{array}$ & Manufacturer \\
\hline $\begin{array}{l}\text { Sulfentrazone } \\
\text { Imazethapyr }\end{array}$ & + & Authority Assist & $480 \mathrm{~g} \mathrm{~L}^{-1}$ & 422 & $\begin{array}{l}\text { FMC Corporation, Philadelphia, PA } \\
19103\end{array}$ \\
\hline $\begin{array}{l}\text { Sulfentrazone } \\
\text { Chloransulam methyl }\end{array}$ & + & Authority First & $621 \mathrm{~g} \mathrm{~kg}^{-1}$ & 315 & $\begin{array}{l}\text { Monsanto Company, } 800 \text { North Lindberg } \\
\text { Ave., St. Louis, Mo }\end{array}$ \\
\hline $\begin{array}{l}\text { Sulfentrazone } \\
\text { Metribuzin }\end{array}$ & + & Authority MTZ & $450 \mathrm{~g} \mathrm{~kg}^{-1}$ & 567 & FMC Corporation \\
\hline $\begin{array}{l}\text { Sulfentrazone } \\
\text { Chlorimuron ethyl }\end{array}$ & + & Authority XL & $700 \mathrm{~g} \mathrm{~kg}^{-1}$ & 343 & FMC Corporation \\
\hline Clomazone & & Command 3ME & $360 \mathrm{~g} \mathrm{~L}^{-1}$ & 840 & FMC Corporation \\
\hline $\begin{array}{l}\text { Chlorimuron methyl } \\
\text { Flumioxazin } \\
\text { Thifensulfuron }\end{array}$ & $\begin{array}{l}+ \\
+\end{array}$ & Enlite & $479 \mathrm{~g} \mathrm{~kg}^{-1}$ & 94 & $\begin{array}{l}\text { DuPont Crop Protection, P. Box } 80705 \\
\text { CRP 705/L1S11, Wilmington, DE } \\
\text { 19880-0705. }\end{array}$ \\
\hline $\begin{array}{l}\text { Flumioxazin } \\
\text { Cloransulam }\end{array}$ & + & Gangster co pack & $\begin{array}{l}510 \mathrm{~g} \mathrm{~kg}^{-1}+ \\
840 \mathrm{~g} \mathrm{~kg}^{-1}\end{array}$ & $107+35.3$ & $\begin{array}{l}\text { Valent USA Corporation, Walnut Creeks, } \\
\text { CA } 94596\end{array}$ \\
\hline Alachlor & & Intrro & $480 \mathrm{~g} \mathrm{~L}^{-1}$ & 2,800 & Monsanto Company \\
\hline $\begin{array}{l}\text { Saflufenacil } \\
\text { Imazethapyr }\end{array}$ & + & Optill & $680 \mathrm{~g} \mathrm{~kg}^{-1}$ & 95 & $\begin{array}{l}\text { BASF Corporation, } 26 \text { Davis Drive, } \\
\text { Research Triangle Park, NC } 27709\end{array}$ \\
\hline $\begin{array}{l}\text { S-metolachlor } \\
\text { Fomesafen }\end{array}$ & + & Prefix & $566 \mathrm{~g} \mathrm{~kg}^{-1}$ & 1,490 & $\begin{array}{l}\text { Syngenta Crop Protection, Inc. } \\
\text { Greensboro, NC } 27419\end{array}$ \\
\hline Pendimethalin & & Prowl $\mathrm{H}_{2} \mathrm{O}$ & $456 \mathrm{~g} \mathrm{~L}^{-1}$ & 1,070 & BASF Ag Products \\
\hline $\begin{array}{l}\text { Pendimethalin } \\
\text { Metribuzin }\end{array}$ & + & $\begin{array}{l}\text { Prowl } \mathrm{H}_{2} \mathrm{O}+ \\
\text { Sencor } \\
\text { DF/Dimetric }\end{array}$ & $\begin{array}{l}456 \mathrm{~g} \mathrm{~L}^{-1}+750 \\
\mathrm{~g} \mathrm{~kg}^{-1}\end{array}$ & $1,070+420$ & $\begin{array}{l}\text { BASF Ag Products }+ \text { AgriSolutions } \\
31832 \text { Delhi Road Brighton, IL } 62012\end{array}$ \\
\hline Imazethapyr & & Pursuit & $240 \mathrm{~g} \mathrm{~L}^{-1}$ & 70 & BASF Corporation \\
\hline $\begin{array}{l}\text { Imazethapyr } \\
\text { S-metolachlor }\end{array}$ & + & $\begin{array}{l}\text { Pursuit }+ \text { Dual II } \\
\text { Magnum }\end{array}$ & $\begin{array}{l}240 \mathrm{~g} \mathrm{~L} \mathrm{~L}^{-1}+ \\
824 \mathrm{~g} \mathrm{~kg}^{-1}\end{array}$ & $137+1,600$ & $\begin{array}{l}\text { BASF Corporation }+ \text { Syngenta Crop } \\
\text { Protection }\end{array}$ \\
\hline $\begin{array}{l}\text { Imazaquin } \\
+ \text { S-metolachlor }\end{array}$ & & $\begin{array}{l}\text { Scepter }+ \text { Dual II } \\
\text { Magnum }\end{array}$ & $\begin{array}{l}700 \mathrm{~g} \mathrm{~kg}^{-1}+ \\
824 \mathrm{~g} \mathrm{~kg}^{-1}\end{array}$ & $137+1,247$ & $\begin{array}{l}\text { BASF Corporation }+ \text { Syngenta Crop } \\
\text { Protection }\end{array}$ \\
\hline $\begin{array}{l}\text { Metribuzin } \\
\text { S-metolachlor }\end{array}$ & + & $\begin{array}{l}\text { Sencor }+ \text { Dual II } \\
\text { Magnum }\end{array}$ & $\begin{array}{l}750 \mathrm{~g} \mathrm{~kg}^{-1}+ \\
824 \mathrm{~g} \mathrm{~kg}^{-1}\end{array}$ & $420+1,070$ & $\begin{array}{l}\text { Bayer Crop Science, Research Triangle } \\
\text { Park, NC } 27709+\text { Syngenta Crop } \\
\text { Protection }\end{array}$ \\
\hline Trifluralin & & Treflan & $480 \mathrm{~g} \mathrm{~L}^{-1}$ & 840 & $\begin{array}{l}\text { Dow AgroSciences, LLC } 9330 \text { Zionsville } \\
\text { Road Indianapolis, IN } 46268\end{array}$ \\
\hline Flumioxazin & & Valor SX & $510 \mathrm{~g} \mathrm{~kg}^{-1}$ & 89 & $\begin{array}{l}\text { Valent U.S.A. Corporation Agricultural } \\
\text { Products }\end{array}$ \\
\hline $\begin{array}{l}\text { Flumioxazin } \\
\text { Chlorimuron-ethyl }\end{array}$ & + & Valor XLT & $597 \mathrm{~g} \mathrm{~kg}^{-1}$ & 113 & $\begin{array}{l}\text { Valent U.S.A. Corporation }+ \text { BASF } \\
\text { Corporation }\end{array}$ \\
\hline
\end{tabular}


Table 2. Details of post-emergence (POST) soybean herbicides used in this study

\begin{tabular}{|c|c|c|c|c|c|c|}
\hline Herbicide & & Trade name & $\begin{array}{l}\text { Rate } \\
\text { g ai ha }{ }^{-1}\end{array}$ & & Manufacturer & Adjuvant $^{\mathrm{a}}$ \\
\hline Quizalofop & & Assure II & 38.6 & & $\begin{array}{l}\text { DuPont Crop Protection, P.O.Box } \\
80705 \text { Wilmington, DE } 19880\end{array}$ & COC $1 \% \mathrm{v} / \mathrm{v}$ \\
\hline Fluthiacet-ethyl & & Cadet & 7.2 & & $\begin{array}{l}\text { FMC Corporation, Philadelphia, PA } \\
19103\end{array}$ & $\begin{array}{l}\text { NIS } 0.25 \% \mathrm{v} / \mathrm{v}+\mathrm{UAN}-28 \% \\
2.34 \mathrm{~L} \mathrm{ha}^{-1}\end{array}$ \\
\hline $\begin{array}{l}\text { Imazethapyr } \\
\text { Glyphosate }\end{array}$ & + & Extreme & 910 & & $\begin{array}{l}\text { Syngenta Crop Protection, Inc. } \\
\text { Greensboro, NC } 27419\end{array}$ & $\begin{array}{l}\text { NIS } 0.125 \% \mathrm{v} / \mathrm{v}+\text { AMS } 2 \% \\
\mathrm{w} / \mathrm{w}\end{array}$ \\
\hline $\begin{array}{l}\text { Fomesafen } \\
\text { Glyphosate }\end{array}$ & + & Flexstar GT & 1,380 & & Syngenta Crop Protection & $\begin{array}{l}\text { NIS } 0.25 \% \mathrm{v} / \mathrm{v}+\text { AMS } 2 \% \\
\mathrm{w} / \mathrm{w}\end{array}$ \\
\hline Fluazifop & & Fusilade DX & 210 & & Syngenta Crop Protection & $\begin{array}{l}\text { NIS } 0.25 \% \mathrm{v} / \mathrm{v}+\mathrm{UAN}-28 \% \\
9.4 \mathrm{~L} \mathrm{ha}^{-1}\end{array}$ \\
\hline $\begin{array}{l}\text { Glyphosate } \\
\text { Imazamox }\end{array}$ & + & $\begin{array}{l}\text { Roundup PowerMAX + } \\
\text { Raptor }\end{array}$ & $\begin{array}{l}1,120 \\
44\end{array}$ & + & $\begin{array}{l}\text { Monsanto Company, } 800 \text { North } \\
\text { Lindberg Ave., St. Louis, Mo }\end{array}$ & $\begin{array}{l}\text { NIS } 0.25 \% \mathrm{v} / \mathrm{v}+\text { AMS } 1.8 \% \\
\mathrm{wt} / \mathrm{wt}\end{array}$ \\
\hline $\begin{array}{l}\text { Glyphosate } \\
\text { Imazaquin }\end{array}$ & + & $\begin{array}{l}\text { Roundup PowerMAX + } \\
\text { Scepter }\end{array}$ & $\begin{array}{l}1,120 \\
76\end{array}$ & + & $\begin{array}{l}\text { Monsanto Company }+ \text { BASF } \\
\text { Corporation, 26 Davis Drive, Research } \\
\text { Triangle Park, NC } 27709\end{array}$ & NIS $0.25 \% \mathrm{v} / \mathrm{v}$ \\
\hline $\begin{array}{l}\text { Glyphosate } \\
\text { Acifluorfen }\end{array}$ & + & $\begin{array}{l}\text { Roundup PowerMAX + } \\
\text { Ultra Blazer }\end{array}$ & $\begin{array}{l}1,120 \\
340\end{array}$ & + & $\begin{array}{l}\text { Monsanto } \text { Company }+ \\
\text { Phosphorus, Inc. } 630 \quad \text { Enited } \\
\text { Business Center, PA 19406 }\end{array}$ & $\begin{array}{l}\text { NIS } 0.25 \% \mathrm{v} / \mathrm{v}+\text { AMS } 2 \% \\
\mathrm{wt} / \mathrm{wt}\end{array}$ \\
\hline Glufosinate & & Liberty $280 \mathrm{SL}$ & 595 & & $\begin{array}{l}\text { Bayer Crop Science, Research Triangle } \\
\text { Park, NC } 27709\end{array}$ & AMS $2 \% \mathrm{wt} / \mathrm{wt}$ \\
\hline Sethoxydim & & Poast Plus & 350 & & BASF Corporation & $\begin{array}{l}\text { COC } 2 \% \mathrm{v} / \mathrm{v}+\text { AMS } 2.8 \% \\
\mathrm{wt} / \mathrm{wt}\end{array}$ \\
\hline Imazamox & & Raptor & 44 & & BASF Corporation & $\begin{array}{l}\text { NIS } 0.25 \% \mathrm{v} / \mathrm{v}+\text { AMS } 1.8 \% \\
\mathrm{wt} / \mathrm{wt}\end{array}$ \\
\hline Clethodim & & Select Max & 136 & & $\begin{array}{l}\text { Valent USA Corporation, Walnut } \\
\text { Creek, CA } 94596\end{array}$ & $\begin{array}{l}\text { NIS } 0.25 \% \mathrm{v} / \mathrm{v}+\text { AMS } 1.8 \% \\
\mathrm{wt} / \mathrm{wt}\end{array}$ \\
\hline $\begin{array}{l}\text { Fenoxaprop } \\
\text { Fluazifop }\end{array}$ & + & Fusion & 135 & & Syngenta Crop Protection & $\begin{array}{l}\text { COC } 0.25 \% \mathrm{v} / \mathrm{v}+\text { AMS } \\
4.5 \% \mathrm{wt} / \mathrm{wt}\end{array}$ \\
\hline $\begin{array}{l}\text { Glyphosate } \\
\text { Chlorimuron-ethy }\end{array}$ & $\begin{array}{c}+ \\
\text { yl }\end{array}$ & $\begin{array}{l}\text { Roundup PowerMAX + } \\
\text { Classic }\end{array}$ & $\begin{array}{l}1,120 \\
5.8\end{array}$ & + & $\begin{array}{l}\text { Monsanto Company }+ \text { DuPont Crop } \\
\text { Protection, P. Box } 80705 \text { CRP } \\
\text { 705/L1S11, Wilmington, DE }\end{array}$ & $\begin{array}{l}\text { NIS } 0.25 \% \mathrm{v} / \mathrm{v}+\text { AMS } 2 \% \\
\mathrm{wt} / \mathrm{wt}\end{array}$ \\
\hline Imazethapyr & & Pursuit & 70 & & BASF Corporation & $\begin{array}{l}\text { NIS } 0.25 \% \mathrm{v} / \mathrm{v}+\text { AMS } 1.8 \% \\
\mathrm{wt} / \mathrm{wt}\end{array}$ \\
\hline Acifluorfen & & Ultra Blazer & 170 & & United Phosphorous Inc. & $\begin{array}{l}\text { NIS } 0.25 \% \mathrm{v} / \mathrm{v}+\text { AMS } 2 \% \\
\mathrm{wt} / \mathrm{wt}\end{array}$ \\
\hline $\begin{array}{l}\text { Imazamox } \\
\text { Acifluorfen }\end{array}$ & + & Raptor + Ultra Blazer & $35+280$ & & $\begin{array}{l}\text { BASF Corporation }+ \text { United } \\
\text { Phosphorous Inc. } \\
+ \text { United phosphorous Inc. }\end{array}$ & $\begin{array}{l}\text { NIS } 0.25 \% \mathrm{v} / \mathrm{v}+\text { AMS } 2 \% \\
\mathrm{wt} / \mathrm{wt}\end{array}$ \\
\hline
\end{tabular}

Note. AMS = ammonium sulfate (DSM chemicals North America Inc., Augusta, GA), COC = crop oil concentrate (Agridex, Helena Chemical Co., Collierville, TN), NIS = nonionic surfactant (Induce, Helena Chemical Co., Collierville, TN), UAN-28 = Urea ammonia nitrate solution 28\% (Sylvite Agri-Services, Ontario, Canada).

\section{Results and Discussion}

\subsection{PRE Herbicide Study}

The two-way interaction of herbicide treatments and volunteer corn type was significant; therefore, data are presented separately. Control of volunteer corn varied among herbicide treatments at $7 \mathrm{~d}$ after treatment (DAT) (Table 3). Control of glufosinate-, glyphosate-, and imidazolinone-resistant volunteer corn was in the range of 9 to $69 \%$, 6 to $58 \%$, and 25 to $69 \%$, respectively, at 7 DAT. However, control was improved in a few herbicide treatments at 21 DAT. For example, although comparable with several other treatments, clomazone provided $\geq$ $90 \%$ control of glufosinate- and imidazolinone-resistant volunteer corn at 21 DAT. Surprisingly, clomazone was not very effective $(<50 \%$ control) on glyphosate-resistant volunteer corn. Cumulative emergence of volunteer 
corn at 21 DAT was comparable with the nontreated control without difference among herbicide treatments, indicating the failure of PRE soybean herbicides to prevent volunteer corn emergence.

Sulfentrazone tank mixes usually resulted in 47 to $75 \%$ control of volunteer corn and was comparable with few other treatments, including clomazone at 21 DAT (Table 3). Volunteer corn biomass reflected similar results with several treatments comparable with the nontreated control that indicated control failure of PRE soybean herbicides. The overall results of the PRE soybean herbicides suggest that with the exception of clomazone for glufosinate- and imidazolinone-resistant volunteer corn, no other herbicide provided economically acceptable control. Based on these greenhouse studies, it is concluded that PRE herbicide is not available for acceptable control of glyphosate-resistant volunteer corn in soybean.

\subsection{POST Herbicide Study}

The three-way interaction of herbicide treatments, volunteer corn type (glufosinate-, glyphosate-, and imidazolinone-resistant), and volunteer corn height was significant. Control of volunteer corn was affected by growth stage and POST soybean herbicides (Table 4). The ACCase-inhibiting herbicides, including clethodim, fenoxaprop plus fluazifop, fluazifop, quizalofop, and sethoxydim, resulted in 48 to $75 \%$ control of glufosinateand glyphosate-resistant volunteer corn at 7 DAT when sprayed at the 2- to 3-leaf stage, and usually were comparable with glyphosate tank-mix treatments. The ACCase inhibitors resulted in 28 to $45 \%$ control of imidazolinone-resistant volunteer corn at 7 DAT; however, control was improved at 28 DAT and resulted in $\geq 96$ $\%$ control, regardless of the resistant trait. Similarly, several studies have reported $>90 \%$ control of volunteer corn with ACCase (Andersen, 1976; Andersen et al., 1982; Andersen \& Geadelmann, 1982; Beckett \& Stroller 1988; Beckett et al., 1992; Marquardt \& Johnson, 2013).

Glyphosate tank mixed with acifluorfen, chlorimuron, imazamox, imazaquin, or imazethapyr usually provided 83 to $91 \%$ and 87 to $98 \%$ control of glufosinate-and imidazolinone-resistant volunteer corn, respectively, and was comparable with an ACCase inhibitor at 28 DAT. Acifluorfen, fluthiacet-ethyl, imazamox, imazethapyr, and imazethapyr plus acifluorfen resulted in poor control $(\leq 57 \%)$ of volunteer corn. Results of volunteer corn control were reflected in biomass. For example, the lowest biomass $\left(\leq 1.2 \mathrm{~g}\right.$ pot $\left.^{-1}\right)$ was recorded with ACCase-inhibitor herbicides and was comparable with glyphosate tank-mix treatments. Fluthiacet-ethyl, imazethapyr, or acifluorfen resulted in the highest biomass that was comparable with the nontreated control and confirmed poor control of volunteer corn in soybean.

The POST soybean herbicides applied at the 5- to 6-leaf stage of volunteer corn resulted in variable response compared with the 2- to 3-leaf stage (Tables 4 and 5). Similarly, Marquardt and Johnson (2013) reported that clethodim applied to $\leq 30 \mathrm{~cm}$-tall volunteer corn provided higher and more consistent control compared to 90 $\mathrm{cm}$-tall plants at $14 \mathrm{DAT}$ at all volunteer corn densities. All herbicide treatments resulted in $<40 \%$ control of volunteer corn at 7 DAT. However, ACCase inhibitors resulted in 85 to $97 \%$ control at 28 DAT. Similarly, several studies demonstrated effective control of volunteer corn with ACCase inhibitors. For example, Andersen et al. (1982) reported $>90 \%$ control of volunteer corn with diclofop. Young and Hart (1997) reported $>90 \%$ control with sethoxydim or quizalofop. Deen et al. (2006) reported that use of a recommended adjuvant significantly improved the effectiveness of ACCase inhibitors, specifically when reduced rates were applied. Glyphosate tank mixed with acifluorfen, chlorimuron, fomesafen, imazamox, imazaquin, and imazethapyr resulted in $\leq 71 \%$ control of volunteer corn, regardless of resistant trait. The lowest volunteer corn biomass was usually recorded with ACCase inhibitors confirming results of visual control estimates at 28 DAT.

Results of the PRE soybean herbicide study revealed that clomazone resulted in $>90 \%$ control of glufosinateand imidazolinone-resistant volunteer corn, but $<50 \%$ control of glyphosate-resistant volunteer corn. A predominant number of corn hybrids planted in the Midwestern United States are glyphosate-resistant, and the occurrence of glyphosate-resistant volunteer corn is more widely distributed compared to glufosinate- and imidazolinone-resistant volunteer corn. In this study, PRE or POST application of imidazolinones resulted in poor control of volunteer corn. In contrast, Young and Hart (1997) reported 70 and 83\% control of volunteer corn with imazaquin and imazethapyr plus imazaquin in soybean. More research is required to identify a PRE herbicide with excellent efficacy for volunteer corn control, soybean selectivity as well as to better understand the natural range in tolerance of volunteer corn lines to herbicides.

Overall results suggest that volunteer corn can be effectively controlled with ACCase inhibitors regardless of herbicide-resistant trait. The ACCase-inhibiting herbicides were more effective and consistent ( $\geq 96 \%$ control) when applied to 2- to 3-leaf stage volunteer corn compared with the 5- to 6-leaf stage ( $\geq 85 \%$ control). Therefore, it is advisable to control volunteer corn with ACCase inhibitors when they are at the 2- to 3-leaf stage to avoid competition with soybean during the early growth stage. In addition, early season control is recommended from 
an insect resistance management standpoint, if volunteer corn plants also express transgenic Bt traits (Krupke et al., 2009). Repeated application of ACCase inhibitors for the last several years has resulted in the evolution of 44 grass weed species resistant to this herbicide chemistry (Heap, 2014). In fact, resistance to ACCase inhibitors has become the third most frequent type of weed resistance (Kukorelli et al., 2013). Therefore, in the fields with ACCase inhibiting herbicide-resistant weed(s), ACCase inhibitors should be tank-mixed with other herbicides that can effectively control resistant weeds without antagonism. Therefore, growers should adopt an integrated volunteer corn management program that may include tillage, crop rotation, and improved cultural agronomic practices to maximize control and reduce the potential for evolution of herbicide-resistant weeds.

Table 3. Effect of PRE soybean herbicides for the control of glufosinate-, glyphosate-, and imidazolinone-resistant volunteer corn at 7 and 21 DAT, cumulative emergence at 21 DAT, and volunteer corn biomass

\begin{tabular}{|c|c|c|c|c|c|c|c|c|c|c|c|c|c|c|}
\hline \multirow{2}{*}{ Herbicide } & \multirow{2}{*}{\multicolumn{2}{|c|}{ Rate }} & \multicolumn{3}{|c|}{ Control at $7 \mathrm{DAT}^{\mathrm{a}, \mathrm{b}}$} & \multicolumn{3}{|c|}{ Control at $21 \mathrm{DAT}^{\mathrm{a}, \mathrm{b}}$} & \multicolumn{3}{|c|}{ Cumulative emergence $21 \mathrm{DAT}^{\mathrm{b}}$} & \multicolumn{3}{|c|}{ Volunteer corn biomass ${ }^{\mathrm{b}}$} \\
\hline & & & Glufo & Glypho & Imida & Glufo & Glypho & Imida & Glufo & Glypho & Imida & Glufo & Glypho & Imida \\
\hline & $\mathrm{g}$ ai ha- & & $\longrightarrow$ & & $\%$ & & & - & $\longrightarrow$ & $\%$ & - & $\longrightarrow$ & $-g \operatorname{pot}^{-1}$ & - \\
\hline $\begin{array}{l}\text { Nontreated } \\
\text { Control }^{c}\end{array}$ & - & & 0 & 0 & 0 & 0 & 0 & 0 & $90 \mathrm{a}$ & $100 \mathrm{a}$ & $100 \mathrm{a}$ & $3.3 \mathrm{a}$ & $3.5 \mathrm{a}$ & $3 \mathrm{a}$ \\
\hline $\begin{array}{l}\text { Sulfentrazone + } \\
\text { Imazethapyr }\end{array}$ & 422 & & $66 \mathrm{ab}$ & $39 \mathrm{ab}$ & $68 \mathrm{a}$ & $64 a-f$ & $50 \mathrm{abc}$ & $72 \mathrm{abc}$ & $80 \mathrm{a}$ & $100 \mathrm{a}$ & $90 \mathrm{a}$ & $1 \mathrm{bc}$ & $1.6 \mathrm{bc}$ & $0.4 \mathrm{bc}$ \\
\hline $\begin{array}{l}\text { Sulfentrazone + } \\
\text { Chloransulam }\end{array}$ & 315 & & $58 \mathrm{ab}$ & $36 \mathrm{ab}$ & $68 \mathrm{a}$ & $65 a-f$ & $48 \mathrm{a}-\mathrm{d}$ & $64 a-d$ & $90 \mathrm{a}$ & $90 \mathrm{a}$ & $80 \mathrm{a}$ & $0.7 \mathrm{bc}$ & $1 b c$ & $0.5 \mathrm{bc}$ \\
\hline $\begin{array}{l}\text { Sulfentrazone + } \\
\text { Metribuzin }\end{array}$ & 567 & & $69 \mathrm{a}$ & $26 a b$ & $69 \mathrm{a}$ & $70 a-d$ & $31 b-f$ & $75 \mathrm{abc}$ & $90 \mathrm{a}$ & $90 \mathrm{a}$ & $80 \mathrm{a}$ & $0.7 \mathrm{bc}$ & $1.1 \mathrm{bc}$ & $0.5 \mathrm{bc}$ \\
\hline $\begin{array}{l}\text { Sulfentrazone + } \\
\text { Chlorimuron }\end{array}$ & 343 & & $55 \mathrm{ab}$ & $35 \mathrm{ab}$ & $66 a b c$ & $68 \mathrm{a}-\mathrm{e}$ & $52 \mathrm{abc}$ & $64 a-d$ & $90 \mathrm{a}$ & $100 \mathrm{a}$ & $70 \mathrm{ab}$ & $0.5 \mathrm{bc}$ & $1 b c$ & $0.3 \mathrm{c}$ \\
\hline Clomazone & 840 & & $50 \mathrm{ab}$ & $16 \mathrm{ab}$ & $68 \mathrm{a}$ & $92 \mathrm{a}$ & $47 \mathrm{a}-\mathrm{e}$ & $90 \mathrm{a}$ & $90 \mathrm{a}$ & $90 \mathrm{a}$ & $60 \mathrm{ab}$ & $0.8 \mathrm{bc}$ & $1.1 \mathrm{bc}$ & $0.4 \mathrm{bc}$ \\
\hline $\begin{array}{l}\text { Chlorimuron + } \\
\text { Flumioxazin + } \\
\text { Thifensulfuron }\end{array}$ & 94 & & $32 \mathrm{ab}$ & $3 b$ & $29 a-e$ & $4 j$ & $6 \mathrm{f}$ & $3 \mathrm{f}$ & $90 \mathrm{a}$ & $100 \mathrm{a}$ & $60 \mathrm{ab}$ & $2.5 \mathrm{abc}$ & $2.3 \mathrm{abc}$ & $1.2 \mathrm{abc}$ \\
\hline $\begin{array}{l}\text { Flumioxazin + } \\
\text { Cloransulam }\end{array}$ & $\begin{array}{l}107 \\
35.3\end{array}$ & + & $43 \mathrm{ab}$ & $6 \mathrm{ab}$ & $58 \mathrm{a}-\mathrm{e}$ & 61a-g & $23 c-f$ & $67 a-d$ & $90 \mathrm{a}$ & $100 \mathrm{a}$ & $70 \mathrm{ab}$ & $0.6 \mathrm{bc}$ & $1.7 \mathrm{abc}$ & $0.5 \mathrm{bc}$ \\
\hline Alachlor & 2,800 & & $44 \mathrm{ab}$ & $8 \mathrm{ab}$ & $39 a-e$ & $22 \mathrm{~g}-\mathrm{j}$ & $4 \mathrm{f}$ & $4 f$ & $80 \mathrm{a}$ & $90 \mathrm{a}$ & $60 \mathrm{ab}$ & $1.9 \mathrm{abc}$ & $2.6 \mathrm{abc}$ & $1.2 \mathrm{abc}$ \\
\hline $\begin{array}{l}\text { Saflufenacil }+ \\
\text { Imazethapyr }\end{array}$ & 95 & & $9 \mathrm{ab}$ & $1 \mathrm{~b}$ & $29 a-e$ & $26 f-j$ & 1f & $12 \mathrm{ef}$ & $90 \mathrm{a}$ & $100 \mathrm{a}$ & $60 \mathrm{ab}$ & $1.6 a b c$ & $2.7 \mathrm{abc}$ & $1.4 \mathrm{abc}$ \\
\hline $\begin{array}{l}\text { S-metolachlor + } \\
\text { Fomesafen }\end{array}$ & 1,490 & & $51 \mathrm{ab}$ & $29 \mathrm{ab}$ & $75 \mathrm{a}$ & $41 \mathrm{c}-\mathrm{j}$ & $13 c-f$ & $81 \mathrm{abc}$ & $80 \mathrm{a}$ & $100 \mathrm{a}$ & $60 \mathrm{ab}$ & $1.3 \mathrm{abc}$ & $2.1 \mathrm{abc}$ & $0.3 \mathrm{c}$ \\
\hline Pendimethalin & 1,070 & & $24 \mathrm{ab}$ & $14 \mathrm{ab}$ & $5 \mathrm{cde}$ & $19 \mathrm{~h}-\mathrm{j}$ & $2 f$ & $1 \mathrm{f}$ & $80 \mathrm{a}$ & $70 \mathrm{a}$ & $70 \mathrm{ab}$ & $2.5 \mathrm{abc}$ & $1.9 \mathrm{abc}$ & $1.9 \mathrm{abc}$ \\
\hline $\begin{array}{l}\text { Pendimethalin } \\
+ \text { Metribuzin }\end{array}$ & $\begin{array}{l}1,070 \\
420\end{array}$ & + & $38 \mathrm{ab}$ & $16 \mathrm{ab}$ & 21a-e & $58 \mathrm{a}-\mathrm{h}$ & $14 c-f$ & $30 \mathrm{def}$ & $90 \mathrm{a}$ & $90 \mathrm{a}$ & $70 \mathrm{ab}$ & $1.5 \mathrm{abc}$ & $1.5 \mathrm{abc}$ & $0.8 \mathrm{bc}$ \\
\hline Imazethapyr & 70 & & $13 \mathrm{ab}$ & $4 b$ & 4cde & $29 e-j$ & $8 \mathrm{def}$ & $1 \mathrm{f}$ & $90 \mathrm{a}$ & $100 \mathrm{a}$ & $80 \mathrm{a}$ & $2.1 \mathrm{abc}$ & $2.1 \mathrm{abc}$ & $2.2 \mathrm{abc}$ \\
\hline $\begin{array}{l}\text { Imazethapyr + } \\
\text { S-metolachlor }\end{array}$ & $\begin{array}{l}137 \\
1,600\end{array}$ & + & $4 b$ & $0.5 b$ & $6 \mathrm{~b}-\mathrm{e}$ & $8 \mathrm{ij}$ & $8 \mathrm{ef}$ & 1f & $90 \mathrm{a}$ & $80 \mathrm{a}$ & $60 \mathrm{ab}$ & $2 a b c$ & $2.2 \mathrm{abc}$ & $1.3 \mathrm{abc}$ \\
\hline $\begin{array}{l}\text { Imazaquin }+ \\
\text { S-metolachlor }\end{array}$ & $\begin{array}{l}137 \\
1,247\end{array}$ & + & $36 \mathrm{ab}$ & $18 \mathrm{ab}$ & 3de & 74abc & $70 \mathrm{ab}$ & $3 \mathrm{f}$ & $70 \mathrm{a}$ & $90 \mathrm{a}$ & $70 \mathrm{ab}$ & $0.6 \mathrm{bc}$ & $0.6 \mathrm{c}$ & $1.4 \mathrm{abc}$ \\
\hline $\begin{array}{l}\text { Metribuzin + } \\
\text { S-metolachlor }\end{array}$ & $\begin{array}{l}420 \\
1,070\end{array}$ & + & $6 \mathrm{~b}$ & $0.5 b$ & $25 a-e$ & $47 b-i$ & $6 \mathrm{f}$ & $32 \mathrm{def}$ & $90 \mathrm{a}$ & $90 \mathrm{a}$ & $60 \mathrm{ab}$ & $1 b c$ & $2 a b c$ & $0.5 \mathrm{bc}$ \\
\hline Trifluralin & 840 & & $5 b$ & $0.5 \mathrm{~b}$ & $3 \mathrm{e}$ & $1 \mathrm{j}$ & $6 \mathrm{f}$ & $1 \mathrm{f}$ & $80 \mathrm{a}$ & $100 \mathrm{a}$ & $80 \mathrm{a}$ & $2.6 \mathrm{ab}$ & $3 a b$ & $2.5 \mathrm{ab}$ \\
\hline Flumioxazin & 89 & & $45 \mathrm{ab}$ & $16 \mathrm{ab}$ & $39 a-e$ & $40 c-j$ & $14 c-f$ & $49 b-e$ & $90 \mathrm{a}$ & $90 \mathrm{a}$ & $70 \mathrm{ab}$ & $1.6 \mathrm{abc}$ & $2.2 \mathrm{abc}$ & $1.3 \mathrm{abc}$ \\
\hline $\begin{array}{l}\text { Flumioxazin + } \\
\text { Chlorimuron }\end{array}$ & 113 & & $28 \mathrm{ab}$ & $11 \mathrm{ab}$ & $29 a-e$ & $31 \mathrm{~d}-\mathrm{j}$ & $21 c-f$ & $44 \mathrm{cde}$ & $90 \mathrm{a}$ & $80 \mathrm{a}$ & $60 \mathrm{ab}$ & $1.3 \mathrm{abc}$ & $1.7 \mathrm{abc}$ & $0.6 \mathrm{bc}$ \\
\hline
\end{tabular}

Glufo = glufosinate-resistant, Glypho = glyphosate-resistant, Imida = imidazolione-resistant.

${ }^{a}$ The data of visual control estimates were arc-sine square-root transformed before analysis; however, data presented are the means of actual values for comparison based on interpretation from the transformed data.

b Means within columns with no common letter(s) are significantly different according to Tukey-Kramer's pairwise comparison test at $\mathrm{P} \leq 0.05$.

${ }^{\mathrm{c}}$ Visual estimates of nontreated control $(0 \%)$ are not included in analysis. 
Table 4. Effect of POST soybean herbicides for the control of 2- to 3-leaf stage glufosinate-, glyphosate-, and imidazolinone-resistant volunteer corn at 7 and 28 DAT and volunteer corn biomass

\begin{tabular}{|c|c|c|c|c|c|c|c|c|c|c|}
\hline \multirow{2}{*}{ Herbicide } & \multirow{2}{*}{ Rate } & \multicolumn{3}{|c|}{ Control at $7 \mathrm{DAT}^{\mathrm{a}, \mathrm{b}}$} & \multicolumn{3}{|c|}{ Control at $28 \mathrm{DAT}^{\mathrm{a}, \mathrm{b}}$} & \multicolumn{3}{|c|}{ Volunteer corn biomass ${ }^{\mathrm{b}}$} \\
\hline & & Glufo & Glypho & Imida & Glufo & Glypho & Imida & Glufo & Glypho & Imida \\
\hline & $\mathrm{g}$ ae or ai ha ${ }^{-1}$ & \multicolumn{6}{|c|}{$\%$} & \multicolumn{3}{|c|}{$-\mathrm{g} \mathrm{pot}^{-1}$} \\
\hline Nontreated Control $^{\mathrm{c}}$ & - & 0 & 0 & 0 & 0 & 0 & 0 & $4 \mathrm{a}$ & $4 \mathrm{a}$ & $4 \mathrm{a}$ \\
\hline Quizalofop & 38.6 & $63 a b c$ & $64 a b c$ & $39 \mathrm{bcd}$ & $99 \mathrm{a}$ & $99 \mathrm{a}$ & $99 \mathrm{a}$ & $1 \mathrm{~b}$ & $1 \mathrm{~b}$ & $1 \mathrm{~d}$ \\
\hline Fluthiacet-ethyl & 7.2 & $33 \mathrm{def}$ & $32 \mathrm{def}$ & $28 \mathrm{cde}$ & $12 \mathrm{~d}$ & $11 \mathrm{ef}$ & $11 \mathrm{ef}$ & $4 \mathrm{a}$ & $4 \mathrm{a}$ & $4 \mathrm{a}$ \\
\hline Imazethapyr + Glyphosate & 910 & $47 b-e$ & $47 \mathrm{~b}-\mathrm{e}$ & $68 \mathrm{a}$ & $85 \mathrm{ab}$ & $53 \mathrm{c}$ & $94 \mathrm{ab}$ & $2 b$ & $2 b$ & $0.7 \mathrm{~d}$ \\
\hline Fomesafen + Glyphosate & 1,380 & $57 \mathrm{a}-\mathrm{d}$ & $57 a-d$ & $57 \mathrm{ab}$ & $70 \mathrm{~b}$ & $56 \mathrm{c}$ & $80 \mathrm{~b}$ & $2 b$ & $2 b$ & $1.2 \mathrm{~cd}$ \\
\hline Fluazifop & 210 & $75 \mathrm{a}$ & $75 \mathrm{a}$ & $45 \mathrm{ab}$ & $99 \mathrm{a}$ & $99 a$ & $99 a$ & $1 \mathrm{~b}$ & $1 \mathrm{~b}$ & $1 \mathrm{~d}$ \\
\hline Glyphosate + Imazamox & $1,120+44$ & $72 \mathrm{ab}$ & $71 \mathrm{ab}$ & $65 \mathrm{a}$ & $91 \mathrm{ab}$ & $65 \mathrm{~b}$ & $95 \mathrm{ab}$ & $1 \mathrm{~b}$ & $1 \mathrm{~b}$ & $1 \mathrm{~d}$ \\
\hline Glyphosate + Imazaquin & $1,120+76$ & $49 a-e$ & $48 \mathrm{a}-\mathrm{e}$ & $55 \mathrm{ab}$ & $85 \mathrm{ab}$ & $59 \mathrm{c}$ & $91 \mathrm{ab}$ & $1 b$ & $1 \mathrm{~b}$ & $1 \mathrm{~d}$ \\
\hline Glyphosate + Acifluorfen & $1,120+340$ & $58 \mathrm{a}-\mathrm{d}$ & $57 \mathrm{a}-\mathrm{d}$ & $60 \mathrm{ab}$ & $83 a b$ & $53 \mathrm{c}$ & $87 \mathrm{ab}$ & $1.5 \mathrm{~b}$ & $1 b$ & $1 \mathrm{~d}$ \\
\hline Glufosinate & 595 & $23 \mathrm{ef}$ & $25 \mathrm{ef}$ & $17 \mathrm{def}$ & $12 \mathrm{~d}$ & $65 \mathrm{~b}$ & $21 \mathrm{cde}$ & $4 \mathrm{a}$ & $2 b$ & $3 a b$ \\
\hline Sethoxydim & 350 & $70 \mathrm{ab}$ & $69 \mathrm{ab}$ & $37 \mathrm{bcd}$ & $97 \mathrm{a}$ & $97 \mathrm{a}$ & $96 a b$ & $1 \mathrm{~b}$ & $1 \mathrm{~b}$ & $1.2 \mathrm{~cd}$ \\
\hline Imazamox & 44 & 31 def & 30def & $9 \mathrm{ef}$ & $57 \mathrm{c}$ & $57 \mathrm{c}$ & $31 \mathrm{c}$ & $2 b$ & $2 b$ & $2 b c$ \\
\hline Clethodim & 136 & $74 \mathrm{ab}$ & $72 \mathrm{ab}$ & $45 \mathrm{abc}$ & $99 \mathrm{a}$ & $99 \mathrm{a}$ & $99 \mathrm{a}$ & $1 \mathrm{~b}$ & $1 \mathrm{~b}$ & $1 \mathrm{~d}$ \\
\hline Fenoxaprop + Fluazifop & 135 & $48 \mathrm{a}-\mathrm{e}$ & $50 \mathrm{a}-\mathrm{e}$ & $28 \mathrm{cde}$ & $98 \mathrm{a}$ & $98 \mathrm{a}$ & $99 \mathrm{a}$ & $1 \mathrm{~b}$ & $1 \mathrm{~b}$ & $1 \mathrm{~d}$ \\
\hline Glyphosate + Chlorimuron-ethyl & $1,120+5.8$ & $51 \mathrm{a}-\mathrm{d}$ & $52 \mathrm{a}-\mathrm{d}$ & $58 \mathrm{ab}$ & $64 \mathrm{~b}$ & $64 \mathrm{~b}$ & $98 \mathrm{a}$ & $1 \mathrm{~b}$ & $2 b$ & $2 \mathrm{bc}$ \\
\hline Imazethapyr & 70 & $5 \mathrm{f}$ & $7 f$ & $2 f$ & $1 \mathrm{~d}$ & $1 \mathrm{~d}$ & 1f & $4 \mathrm{a}$ & $4 a$ & $3 a b$ \\
\hline Acifluorfen & 170 & $32 \mathrm{def}$ & 30def & $28 \mathrm{cde}$ & $10 \mathrm{~d}$ & $10 \mathrm{~d}$ & $13 \mathrm{def}$ & $4 \mathrm{a}$ & $4 \mathrm{a}$ & $4 \mathrm{a}$ \\
\hline Imazamox + Acifluorfen & $35+280$ & $38 \mathrm{cde}$ & $36 \mathrm{~cd}$ & $36 \mathrm{bcd}$ & $50 \mathrm{c}$ & $51 \mathrm{c}$ & $30 \mathrm{~cd}$ & $2 b$ & $2 b$ & $3 \mathrm{ab}$ \\
\hline
\end{tabular}

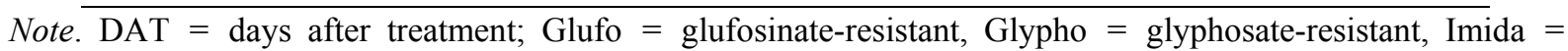
imidazolione-resistant.

${ }^{a}$ The data of visual control estimates were arc-sine square-root transformed before analysis; however, data presented are the means of actual values for comparison based on interpretation from the transformed data.

${ }^{b}$ Means within columns with no common letter(s) are significantly different according to Tukey-Kramer's pairwise comparison test at $\mathrm{P} \leq 0.05$. 
Table 5. Effect of POST soybean herbicides for control of 5- to 6-leaf stage glufosinate-, glyphosate-, and imidazolinone-resistant volunteer corn at 7 and 28 DAT and volunteer corn biomass

\begin{tabular}{|c|c|c|c|c|c|c|c|c|c|c|}
\hline \multirow[b]{2}{*}{ Herbicide } & \multirow[b]{2}{*}{ Rate } & \multicolumn{3}{|c|}{ Control at $7 \mathrm{DAT}^{\mathrm{a}, \mathrm{b}}$} & \multicolumn{3}{|c|}{ Control at $28 \mathrm{DAT}^{\mathrm{a}, \mathrm{b}}$} & \multicolumn{3}{|c|}{ Volunteer corn biomass ${ }^{\mathrm{b}}$} \\
\hline & & Glufo & Glypho & Imida & Glufo & Glypho & Imida & Glufo & Glypho & Imida \\
\hline & $\mathrm{g}$ ae or ai ha ${ }^{-1}$ & \multicolumn{6}{|c|}{ 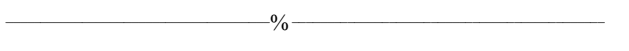 } & \multicolumn{3}{|c|}{$-\operatorname{gpot}^{-1}$} \\
\hline Nontreated control $^{\mathrm{c}}$ & - & 0 & 0 & 0 & 0 & 0 & 0 & $6 \mathrm{ab}$ & $6 \mathrm{ab}$ & $6 \mathrm{ab}$ \\
\hline Quizalofop & 38.6 & $8 \mathrm{de}$ & $8 \mathrm{de}$ & $6 \mathrm{~g}$ & $97 \mathrm{a}$ & $97 \mathrm{a}$ & $97 \mathrm{a}$ & $2 \mathrm{f}$ & $2 \mathrm{f}$ & $3 \mathrm{ef}$ \\
\hline Fluthiacet-ethyl & 7.2 & $3 \mathrm{e}$ & $3 \mathrm{e}$ & $3 \mathrm{~g}$ & $3 \mathrm{f}$ & $3 \mathrm{f}$ & $4 \mathrm{e}$ & $6 \mathrm{ab}$ & $6 \mathrm{ab}$ & $7 \mathrm{a}$ \\
\hline Imazerthapyr + Glyphosate & 910 & $19 b-e$ & $19 \mathrm{~b}-\mathrm{e}$ & $23 b-f$ & 34 cde & $34 \mathrm{cde}$ & $60 \mathrm{~cd}$ & $4.5 \mathrm{~b}-\mathrm{e}$ & $4.5 \mathrm{~b}-\mathrm{e}$ & $4 \mathrm{def}$ \\
\hline Fomesafen + Glyphosate & 1,380 & $24 a-d$ & $24 a-d$ & $30 a-d$ & $44 \mathrm{~cd}$ & $45 \mathrm{~cd}$ & $55 \mathrm{~d}$ & $4 b-f$ & $4 b-f$ & $4.8 \mathrm{~b}-\mathrm{e}$ \\
\hline Fluazifop & 210 & $12 \mathrm{cde}$ & $12 \mathrm{cde}$ & $13 \mathrm{efg}$ & $98 \mathrm{a}$ & $97 \mathrm{a}$ & $95 \mathrm{a}$ & $2 \mathrm{f}$ & $2 f$ & $2.8 \mathrm{f}$ \\
\hline Glyphosate + Imazamox & $1,120+44$ & $38 \mathrm{a}$ & $38 \mathrm{a}$ & $42 \mathrm{a}$ & $69 \mathrm{bc}$ & $63 \mathrm{bc}$ & $71 \mathrm{a}-\mathrm{d}$ & $2.5 \mathrm{f}$ & $3 \mathrm{ef}$ & $2.8 \mathrm{f}$ \\
\hline Glyphosate + Imazaquin & $1120+76$ & $24 a-d$ & $24 a-d$ & $27 \mathrm{a}-\mathrm{e}$ & $58 \mathrm{~cd}$ & $43 \mathrm{~cd}$ & $66 \mathrm{bcd}$ & $4 b-f$ & $4 c-f$ & $3.3 \mathrm{def}$ \\
\hline Glyphosate + Acifluorfen & $1,120+340$ & $28 \mathrm{abc}$ & $28 \mathrm{abc}$ & $36 \mathrm{ab}$ & $55 \mathrm{~cd}$ & $38 \mathrm{~cd}$ & $59 \mathrm{~cd}$ & $4.5 \mathrm{~b}-\mathrm{e}$ & $4.5 \mathrm{~b}-\mathrm{e}$ & $4 c-f$ \\
\hline Glufosinate & 595 & $3 \mathrm{e}$ & $3 \mathrm{e}$ & $9 \mathrm{fg}$ & $8 \mathrm{ef}$ & $8 \mathrm{ef}$ & $17 \mathrm{e}$ & $5 \mathrm{a}-\mathrm{d}$ & $5 \mathrm{a}-\mathrm{d}$ & $5 \mathrm{a}-\mathrm{e}$ \\
\hline Sethoxydim & 350 & $16 \mathrm{~b}-\mathrm{e}$ & $16 \mathrm{~b}-\mathrm{e}$ & $14 \mathrm{~d}-\mathrm{g}$ & $87 \mathrm{ab}$ & $85 \mathrm{ab}$ & $87 \mathrm{ab}$ & $2.7 \mathrm{ef}$ & 2.6ef & $3 \mathrm{ef}$ \\
\hline Imazamox & 44 & $3 \mathrm{e}$ & $3 \mathrm{e}$ & $3 \mathrm{~g}$ & $21 \mathrm{def}$ & $21 \mathrm{def}$ & $15 \mathrm{e}$ & $5.5 \mathrm{abc}$ & $5.5 \mathrm{abc}$ & $6 a b c$ \\
\hline Clethodim & 136 & $18 \mathrm{~b}-\mathrm{e}$ & $18 \mathrm{~b}-\mathrm{e}$ & $17 \mathrm{c}-\mathrm{g}$ & $89 \mathrm{a}$ & $88 \mathrm{a}$ & $88 \mathrm{ab}$ & $3 \mathrm{def}$ & $3 \mathrm{def}$ & $3 \mathrm{ef}$ \\
\hline Fenoxaprop + Fluazifop & 135 & $16 \mathrm{~b}-\mathrm{e}$ & $16 \mathrm{~b}-\mathrm{e}$ & $13 \mathrm{efg}$ & $87 \mathrm{ab}$ & $86 \mathrm{ab}$ & $86 a b$ & $3 \mathrm{def}$ & $3 \mathrm{def}$ & $2.7 \mathrm{f}$ \\
\hline $\begin{array}{l}\text { Glyphosate } \\
\text { Chlorimuron-ethyl }\end{array}$ & $1,120+5.8$ & $33 \mathrm{ab}$ & $33 \mathrm{ab}$ & $33 a b c$ & $50 \mathrm{~cd}$ & $44 \mathrm{~cd}$ & $65 \mathrm{bcd}$ & $4.5 \mathrm{~b}-\mathrm{e}$ & $4.5 \mathrm{~b}-\mathrm{e}$ & $3 e f$ \\
\hline Imazethapyr & 70 & $2 \mathrm{e}$ & $2 \mathrm{e}$ & $1 \mathrm{~g}$ & $3 \mathrm{f}$ & $3 \mathrm{f}$ & $1 \mathrm{e}$ & $6 \mathrm{ab}$ & $6 \mathrm{ab}$ & $5.8 \mathrm{abc}$ \\
\hline Acifluorfen & 170 & $4 \mathrm{e}$ & $4 \mathrm{e}$ & $2 \mathrm{~g}$ & $4 \mathrm{f}$ & $3 \mathrm{f}$ & $4 e$ & $6 a b$ & $6 a b$ & $5.8 \mathrm{abc}$ \\
\hline Imazamox + Acifluorfen & $35+280$ & $8 \mathrm{de}$ & $8 \mathrm{de}$ & $7 \mathrm{fg}$ & $9 \mathrm{ef}$ & $8 \mathrm{f}$ & $10 \mathrm{e}$ & $6 a b$ & $6 a b$ & $5 \mathrm{a}-\mathrm{d}$ \\
\hline
\end{tabular}

Note. DAT $=$ days after treatment; Glufo $=$ glufosinate-resistant, Glypho $=$ glyphosate-resistant, Imida $=$ imidazolione-resistant.

a The data of visual control estimates were arc-sine square-root transformed before analysis; however, data presented are the means of actual values for comparison based on interpretation from the transformed data.

b Means within columns with no common letter(s) are significantly different according to Tukey-Kramer's pairwise comparison test at $\mathrm{P} \leq 0.05$.

${ }^{\mathrm{c}}$ Visual estimates of nontreated control $(0 \%)$ are not included in analysis.

\section{References}

Andersen, R. N. (1976). Control of volunteer corn and giant foxtail in soybeans. Weed Sci., 24, 253-256.

Andersen, R. N., \& Geadelmann, J. L. (1982). The effect of parentage on the control of volunteer corn (Zea mays) in soybeans (Glycine max). Weed Sci., 30, 127-131.

Andersen, R. N., Ford, J. H., \& Lueschen, W. E. (1982). Controlling volunteer corn (Zea mays) in soybeans (Glycine max) with diclofop and glyphosate. Weed Sci., 30, 132-136.

Beckett, T. H., \& Stoller, E. W. (1988). Volunteer corn (Zea mays) interference in soybeans (Glycine max). Weed Sci., 36, 159-166.

Beckett, T. H., Stroller, E. W., \& Bode, L. E. (1992). Quizalofop and sethoxydim activity as affected by adjuvants and ammonium fertilizers. Weed Sci., 40, 12-19.

Castle, L. A., Wu, G. S., \& McElroy, D. (2006). Agricultural input traits: past, present and future. Curr Opin Biotechnol., 17, 105-112. http://dx.doi.org/10.1016/j.copbio.2006.01.011 
Dale, J. E. (1981). Control of johnsongrass (Sorghum halepense) and volunteer corn (Zea mays) in soybeans (Glycine max). Weed Sci., 40, 12-19.

Deen, W., Hamill, A., Shropshire, C., Soltani, N., \& Sikkema, P. H. (2006). Control of glyphosate-resistant corn (Zea mays) in glyphosate-resistant soybean (Glycine max). Weed Technol., 20, 261-266. http://dx.doi.org/10.1614/WT-02-128.1

Heap, I. M. (2014). The international survey of herbicide resistant weeds. Retrieved from http://www.weedscience.org

Krupke, C., Marquardt, P. T., Johnson, W. G., Weller, S., \& Conley, S. P. (2009). Volunteer corn presents new challenges for insect resistance management. Agron. J., 101, 797-799. http://dx.doi.org/10.2134/agronj2008.0149Nx

Kukorelli, G., Reisinger, P., \& Pinke, G. (2013). ACCase inhibitor herbicides- selectivity, weed resistance and fitness cost: a review. Int. J. Pest Manag., 59, 165-173. http://dx.doi.org/10.1080/09670874.2013.821212

Malcolm, S., \& Aillery, M. (2009). Growing crops for biofuel has spillover effects. Amber Waves., 7, 10-15.

Marquardt, P. T., \& Johnson, W. G. (2013). Influence of clethodim application timing on control of volunteer corn in soybean. Weed Technol., 27, 645-648. http://dx.doi.org/10.1614/WT-D-12-00188.1

Marquardt, P. T., Krupke, C., \& Johnson, W. G. (2012b). Competition of transgenic volunteer corn with soybean and the effect on western corn rootworm emergence. Weed Sci., 60, 193-198. http://dx.doi.org/10.1614/WS-D-11-00133.1

Marquardt, P. T., Terry, R., Krupke, C. H., \& Johnson, W. G. (2012a). Competitive effects of volunteer corn on hybrid corn growth and yield. Weed Sci., 60, 537-514. http://dx.doi.org/10.1614/WS-D-11-00219.1

Shauck, T. C., \& Smeda, R. J. (2012). Control of glyphosate-resistant corn (Zea mays) with glufosinate or imazethapyr plus imazapyr in a replant situation. Weed Technol., 26, 417-421. http://dx.doi.org/10.1614/WT-D-11-00138.1

Shaw, J. T., Paullus, J. H., \& Luckmann, W. H. (1978). Corn rootworm oviposition in soybeans. Econ Ento., 71, 189-191.

Steckel, L. E., Thompson, M. A., \& Hayes, R. M. (2009). Herbicide options for controlling glyphosate-tolerant corn in a corn replant situation. Weed Technol., 23, 243-246. http://dx.doi.org/10.1614/WT-08-151.1

[USDA] U.S. Department of Agriculture-World Agricultural Supply and Demand Estimates. (2013). Retrieved from http://www.usda.gov/oce/commodity/wasde/index.htm

[USDA-NASS] U.S. Department of Agriculture-National Agriculture Statistical Service. (2010). Acreage Report. Retrieved from http://usda.mannlib.cornell.edu/usda/nass/Acre/2010s/2010/Acre-06-30-2010.pdf_

Wilson, R., Sandell, L. D., Klein, R., \& Bernards, M. (2010). Volunteer corn control. Proceedings of 2010 Crop Production Clinics (pp. 212-215). Lincoln, NE: University of Nebraska-Lincoln Extension.

Young, B. G., \& Hart, S. E. (1997). Control of volunteer sethoxydim-resistant corn (Zea mays) in soybean (Glycine max). Weed Technol., 11, 649-655.

\section{Copyrights}

Copyright for this article is retained by the author(s), with first publication rights granted to the journal.

This is an open-access article distributed under the terms and conditions of the Creative Commons Attribution license (http://creativecommons.org/licenses/by/3.0/). 